\title{
A Bama Minipig Model of Laryngopharyngeal Reflux and the Change of Laryngopharyngeal Mucosal Ultrastructure
}

\author{
Guijian Feng, ${ }^{1 *}$ Zhenyu Zhang, ${ }^{2}$ Chunyan Diao, ${ }^{1}$ Jun Jiang, ${ }^{3}$ Shuying Zheng ${ }^{4}$ and Yulan Liu ${ }^{1 *}$ \\ ${ }^{1}$ Department of Gastroenterology, Peking University People's Hospital, Beijing, China; '2Department of Gastroenterology, Puyang County \\ People's Hospital, Henan, China; ${ }^{3}$ Beijing chuang shi century minipig breeding base, Beijing, China; and ${ }^{4}$ Department of Electron Microscope \\ Laboratory, Peking University People's Hospital, Beijing, China
}

\section{Background/Aims}

To establish an animal model of laryngopharyngeal reflux (LPR) and study the effect of LPR on the laryngopharyngeal mucosal ultrastructure.

\begin{abstract}
Methods
Ten Bama minipigs were randomly divided into control group and stent group. Every pig underwent endoscope, and baseline $\mathrm{pH}$ was monitored for 4 hours at laryngopharynx and distal esophagus, then specimens from laryngopharyngeal mucosa were biopsied. For the control group, these procedures were repeated on the 14th day. In the stent group, a custom-designed esophageal stent suit was implanted into esophagus, laryngopharyngeal and distal esophageal pH monitoring lasted for 2 hours, then stent suit was removed 3 days later. At last, the same procedures were done as the control group on the 14th day. Specimens were observed under transmission electron microscope to measure the intercellular space and desmosome number.

Results

In the control group, there was no laryngopharyngeal reflux on the first day and 14th day. Before the stent was implanted, there was also no laryngopharyngeal reflux in the stent group. In both 2 hours and 14 days after stent implantation, the number of reflux, reflux time, and percentage time of $\mathrm{pH}<4.0$ were significantly increased $(P<0.05)$ in the stent group. There was no difference in intercellular space and desmosomes in the control group between baseline and 14th day. In the stent group, intercellular space of laryngopharyngeal mucosa was significantly increased $(0.37 \mu \mathrm{m}$ vs $0.96 \mu \mathrm{m}, P=0.008)$, and the number of desmosomes was significantly decreased $(20.25$ vs $9.5, P=0.003)$.
\end{abstract}

\section{Conclusions}

A Bama minipig model of LPR was established by implanting a custom-designed stent suit. LPR might destroy the laryngophar

Received: September 30, 2014 Revised: December 23, 2014 Accepted: December 25, 2014

(c) This is an Open Access article distributed under the terms of the Creative Commons Attribution Non-Commercial License (http://creativecommons. org/licenses/by-nc/3.0) which permits unrestricted non-commercial use, distribution, and reproduction in any medium, provided the original work is properly cited.

*Correspondence: Guijian Feng and Yulan Liu are equally responsible for this study.

Guijian Feng, MD

Department of Gastroenterology, Peking University People's Hospital, Beijing 100044, China

Tel: +86-88325388, Fax: +86-68318386, E-mail: fggods@126.com

Yulan Liu, MD

Department of Gastroenterology, Peking University People's Hospital, Beijing 100044, China

Tel: +86-88325559, Fax: +86-68318386, E-mail: liuyulan@pkuph.edu.cn

Financial support: This study was supported by National Natural Science Foundation of China (project No. 812245).

Conflicts of interest: None.

Author contributions: Guijian Feng, planning and conducting the study, collecting and interpreting data, and drafting the manuscript; Zhenyu Zhang, stent implanting; Chunyan Diao, stent implanting; Jun Jiang, minipig breeding; Shuying Zheng, Electron Microscope; and Yulan Liu, planning and conducting the study.

ORCID: Guijian Feng, http://orcid.org/0000-0002-6400-847X. 
yngeal mucosal barrier.

\section{(J Neurogastroenterol Motil 2015;21:182-188)}

\section{Key Words}

Extracellular space; Laryngopharyngeal reflux; Models, animal

\section{Introduction}

Laryngopharyngeal reflux (LPR) was first defined by the American Academy of Otolaryngology-Head and Neck Surgery in 2002. ${ }^{1}$ LPR disease (LPRD) refers to the backflow of stomach contents (eg, acid, bile, pepsinogen, etc) into the laryngopharynx through the upper esophageal sphincter, resulting in injuries to the laryngopharynx, oral cavity, and respiratory tract. It was reported that $4-10 \%$ of patients referred to ear, nose, and throat (ENT) physicians were diagnosed with $\mathrm{LPR}^{2,3}$ and a number of patients with gastroesophageal reflux disease (GERD) in the department of gastroenterology were also identified to be suffering from LPR. ${ }^{4}$ LPRD is usually complicated with chronic pharyngitis, laryngitis, laryngeal carcinoma, bronchial asthma, and sleep apnea syndrome, which can impact the quality of life. ${ }^{5-8}$ Compared with GERD, patients with LPRD had a longer treatment course and higher cost of treatment. Moreover, the incidence of LPR has been increasing over the past few years. Therefore, it would be very important to establish LPR animal model in order to study the mechanism and treatment to LPR.

In order to establish the exact model of LPR, it should be kept in mind that the abnormal dynamic barrier is the initiator of LPR. The gastroesophageal junction constitutes the first barrier against LPR, including lower esophageal sphincter (LES) and phrenic angle. Normal motility and clearing function of esophageal body are the second barriers against LPR. In GERD patients, especially in some patients with reflux esophagitis, esophageal motility was found to be ineffective. ${ }^{9,10}$ Knight et al ${ }^{11}$ also found $73 \%$ patients with LPR symptoms to have esophageal motility problems. Tsutsui et $\mathrm{al}^{12}$ reported that $47.9 \%$ had abnormal esophageal motility among patients with proton pump inhibitor (PPI)-resistant LPRD. The last barrier against LPR is upper esophageal sphincter (UES) in which tonic cricopharyngeal muscle contraction maintains the high pressure of UES. One recent study observed that abnormal UES and esophageal motility associated with impaired bolus clearance in patients with chronic cough were mostly marked in patients with a positive reflux- cough symptom association. ${ }^{13}$

If there is a way to make esophagus lose these barriers, then a new laryngopharyngeal reflux model will be established. Esophageal stent implant is an important method to treat esophageal stenosis with many kinds of stents showing anti reflux function. ${ }^{14-16}$ The stent without anti reflux function is likely to damage barrier function of esophagus and cardia. When a stent is placed under the UES or LES, the function of esophageal body is completely shielded, leading to the induction of LPR. In the present study, we for the first time used a specialized custom stent suit to destroy LES and the barrier of esophageal body, and successfully established a Bama minipig model of LPR. Furthermore, the changes of laryngopharyngeal mucous ultrastructure have not been largely studied. In GERD, intracellular space of esophageal cells is increased, ${ }^{17-19}$ and in LPR with GERD, Park et al ${ }^{20}$ reported increased intracellular space also in esophagus. We have also studied the ultrastructure of laryngopharyngeal mucosa in this model to clarify the pathological mechanism of LPR.

\section{Materials and Methods}

\section{Animals}

Ten male Bama minipigs were purchased from Beijing chuang shi century minipig breeding base. The Bama minipigs were 4-6 months old, the mean weight was $10 \mathrm{~kg}$, and the mean length was $71 \mathrm{~cm}$. Minipigs were maintained in a pathogen-free environment with a 12:12-hour light-dark cycle, temperature $\left(20-22^{\circ} \mathrm{C}\right)$ and humidity (50-60\%) for 1 week prior to experiment in order to adapt to environment. Ten Bama minipigs were randomly divided into control group and stent group. All animal care and procedures were approved by the Animal Care Committee of Beijing University People's Hospital and complied with the Guide for the Care and Use of Laboratory Animals published by the United States National Institutes of Health (NIH publication 80-23, revised 1996). 


\section{$\mathrm{pH}$ Monitoring}

Anesthesia was induced with intramuscular injection of a mixture of $1 \mathrm{mg}$ ketamine and Sumianxin II for each pig. Anesthesia was maintained with Phenobarbital when needed by $1 \mathrm{mg}$ prn. The dose was adjusted according to the depth of anesthesia. Trachea cannula was inserted when the eyelash reflex ceased.

The minipigs were examined using endoscopy (GIF-2T200 and GIF-XP-260N; Olympus Medical Systems Corp, Tokyo, Japan) to determine the distance from esophageal orifice to the incisor tooth and the distance from the cardia dentate line to the incisor tooth. Oropharyngeal electrode was implanted in the esophageal orifice to monitor pharynx $\mathrm{pH}$ using the Restech Dx-pH system (Restech Company, San Diego, CA, USA). The distal esophagus electrode was implanted $5 \mathrm{~cm}$ above the cardia dentate line to monitor esophagus $\mathrm{pH}$ using the Sandhill system (Sandhill Z07-2000B-B monitor; Sandhill Scientific, Highlands Ranch, CO, USA). Endoscope was used to determine the success of implanting electrode. After baseline $\mathrm{pH}$ was monitored for 4 hours, the laryngohypopharynx mucosa specimens were immediately excised and fixed in $4 \%$ formaldehyde solution for electron microscope examination. All the $\mathrm{pH}$ monitoring data were analyzed by the manufacturer's software.

\section{Stent Implantation}

According to the literature, esophageal length of the Bama minipig was about $25 \mathrm{~cm}$. The manufacturer can only provide stent less than $18 \mathrm{~cm}$. Esophageal stent suit was custom-designed by Micro-Tech Co, Ltd (Nanjing, China). The stent suit included 2 stents made from nickel-titanium alloy. The length of each stent was $14 \mathrm{~cm}$, and the diameter was $2.2 \mathrm{~cm}$ and $2.0 \mathrm{~cm}$. The basilar stent had an umbrella in order to be fixed in the gastric cardia and the upper stent had a recycling line at the upper part to be fixed through the nasal cavity, while the body of stents was covered by membrane (Fig. 1). The location of the cardia was determined by endoscope, and the head of the guidewire was put in the antrum of the stomach, then we draw back the endoscope, the stent towveyor entered the stomach and the baseline stent was released. We repeated this procedure to implant another stent. Then the $\mathrm{pH}$ of pharyngolarynx and distal esophagus were monitored for approximately 2 hours. On the third day after surgery, the stent was removed. On the 14th day after surgery, the $\mathrm{pH}$ of pharyngolarynx and distal esophagus was monitored again, and the laryngopharynx mucosa specimens were excised by biopsy forceps simultaneously.

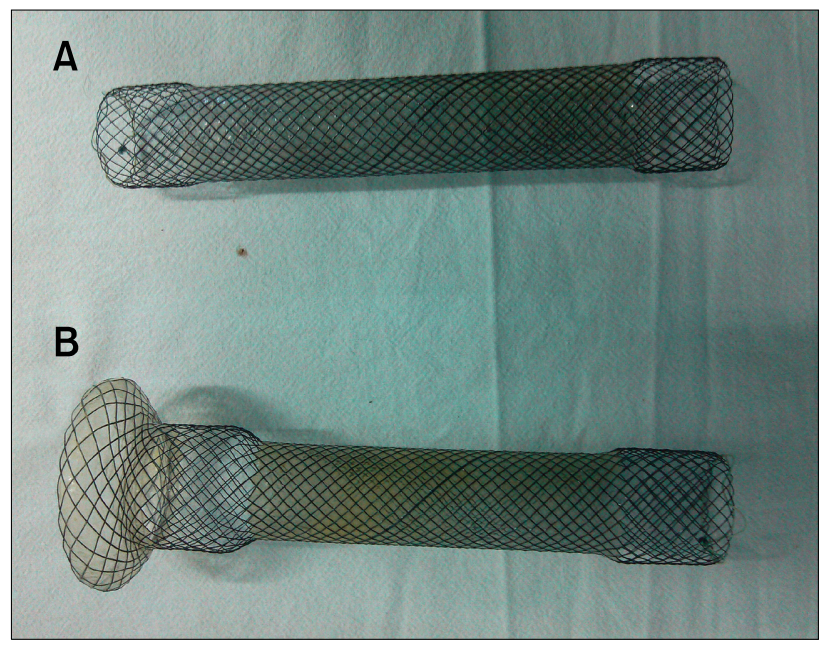

Figure 1. Images of the custom-designed stent suits. (A) The upper stent had a recycling line to be fixed through the nasal cavity and to be removed more easily. (B) The basilar stent had an umbrella in order to be fixed in the gastric cardia.

\section{Transmission Electron Microscope Examination}

Tissue preparation for electron microscopy was according to the previous study. Ten pictures were obtained from each case, one entire cell was selected in each picture, and intercellular space was measured from 10 random directions (vertical to the neighboring cell membrane, and no less than $1 \mu \mathrm{m}$ between each direction) using a transmission electron microscope (Tecani sprit; FEI, Hillsboro, Oregon, USA). The average width of 100 intercellular spaces was considered as the intercellular spaces of one case. The average desmosome number of ten intercellular spaces from 10 pictures was considered as the desmosome number of one case.

\section{Statistical Methods}

Data were expressed as mean \pm standard deviation. Chisquare test, paired samples $t$ test, independent samples $t$ test and one-way ANOVA with repeated measures were used for statistical analysis where appropriate. Analyses were performed by the SPSS software (Statistical Package for the Social Sciences version 18.0; SPSS Inc, Chicago, IL, USA). $P<0.05$ was considered statistically significant.

\section{Results}

There were 10 Bama minipigs enrolled in this procedure, one 


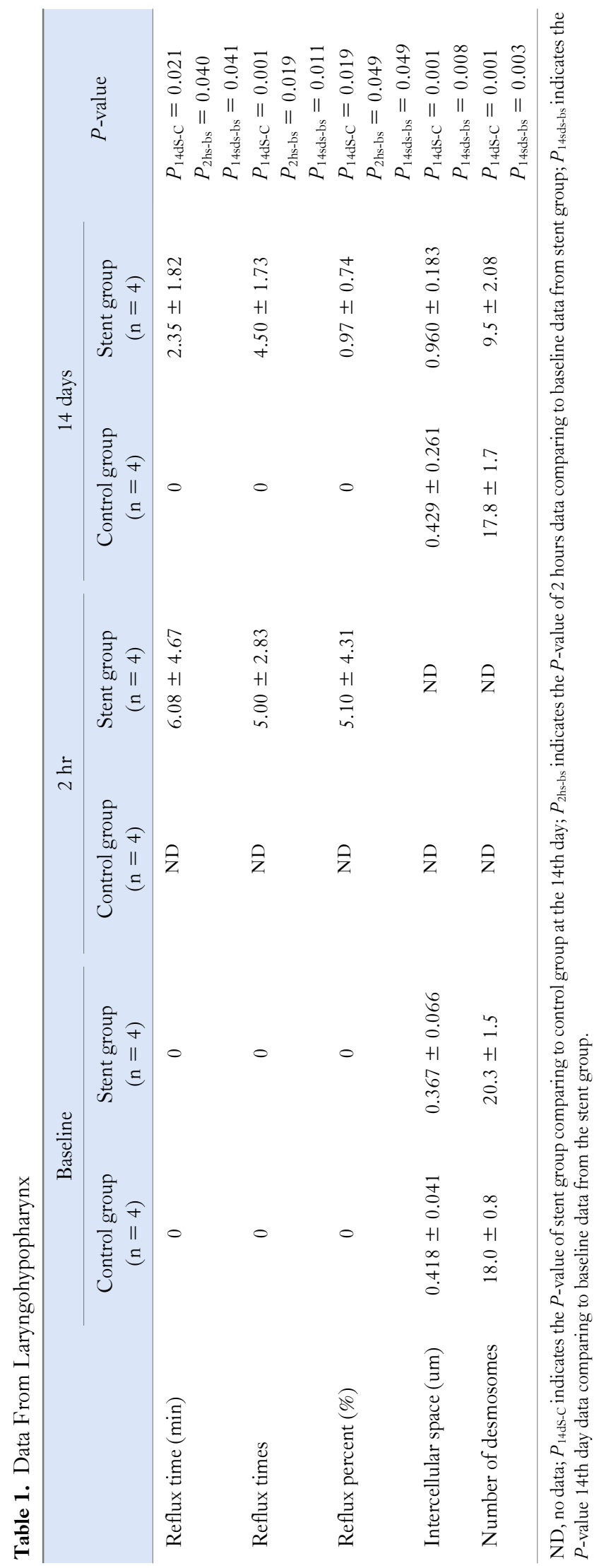

from each of two group died of undetermined choke during the procedure. Because one pig died from each group, the success rates for both the acute and benign LPR model were $80 \%$.

\section{Evaluation of the Laryngopharyngeal Reflux Model}

In the control group, no reflux was observed, as assessed by laryngopharyngeal $\mathrm{pH}$ monitoring on the first and 14th day after surgery (Table 1). Before the stent implantation, there was also no reflux in the stent group. Therefore we concluded that the model of LPR could be considered successful if reflux occurred at least once after stent implantation.

Two hours after stent implantation, the number of reflux events, reflux time, and percentage were significantly higher $(P$ $<0.05)$ in the stent group compared with baseline (Fig. 2). Eleven days after removing the stent (ie, 14 days after stent implantation), there was still reflux. One or more reflux events occurred in every pig, not only 2 hours after stent implantation but also on the 14th day.

\section{Changes in Gastroesophageal Reflux}

In this LPR model, gastroesophageal reflux (GER) was also induced. In the control group, there was no change in GER between the first and 14th days after the procedure (Table 2). However, 2 hours after stent implantation the number of reflux events, reflux time, and percentage were significantly higher $(P$ $<0.05)$ in the stent group compared with the control group.

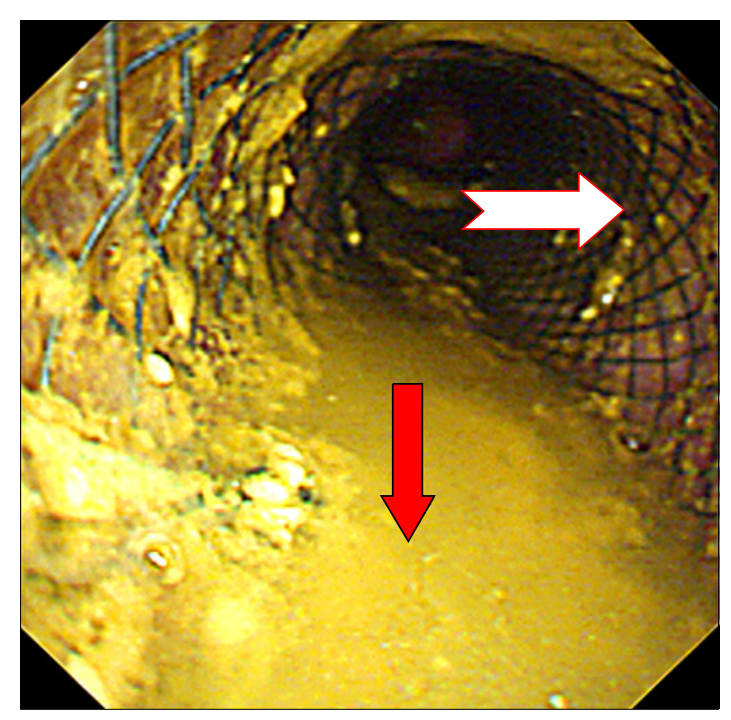

Figure 2. Endoscope examination of the stent in esophagus and reflux. There are stents in esophagus (white arrow), and much of reflux content (red arrow). 
Eleven days after removing the stent (14 days after stent implantation), there was still reflux increasing, and the differences between the control and stent groups were significant $(P<0.05)$ except for acid reflux.

\section{Changes in Intercellular Space and Number of Desmosomes}

In the control group, there were no changes in intercellular space or number of desmosomes between the first day and 14th day after the procedure (Table 1). However, in the stent group the intercellular space was significantly higher on the 14th day $(0.96 \mu \mathrm{m})$ compared with the first day $(0.37 \mu \mathrm{m}, P=0.008)$, and the number of desmosomes were significantly lower (20.25 on the first day, compared with 9.5 on the 14th; $P=0.003$; Fig. 3).

\section{Discussion}

In the present study, a Bama minipig model of LPR was established by implanting a custom-designed stent suit, which was assessed by $\mathrm{pH}$ monitoring at the sites of laryngopharynx and distal esophagus. Intercellular space was significantly increased and the number of desmosomes was significantly decreased in the laryngopharyngeal mucus of LPR model, suggesting that LPR might destroy the laryngopharyngeal mucosal barrier by change the mucosal ultrastructure.

Gill et $\mathrm{al}^{21}$ studied human and non-human laryngeal tissues (mouse, rat, guinea pig, porcine, and rabbit) and found that porcine tissue revealed both respiratory-type and stratified squamous epithelium, as seen in the human larynx. The authors indicated that the porcine larynx was a superior model for research of epithelial damage occurring during LPR. ${ }^{21}$ In the present study, Bama minipigs were used. The miniature pig has been widely accepted as the experimental animal. ${ }^{22-25}$ With its body size being only one-fourth to one-third of an ordinary pig, ${ }^{26}$ it is easy to be operated and thus suitable to establish a LPR model.

Although the pathological mechanisms of LPR and GERD are similar, LPR model was rarely reported, compared to GERD model. There were 2 previous methods to establish GERD animal model: one was endogenous GERD induced by its own gastrointestinal reflux and the other was an exogenous GERD induced by exposure to acidic solutions. In endogenous GERD model, surgical operation was used to change normal gastrointestinal tract anatomy resulting in reflux of gastric juice, duodenal juice, or the mixture. The operation methods included ligation of pylorus and proximal small bowel, destruction of cardia, and dif-

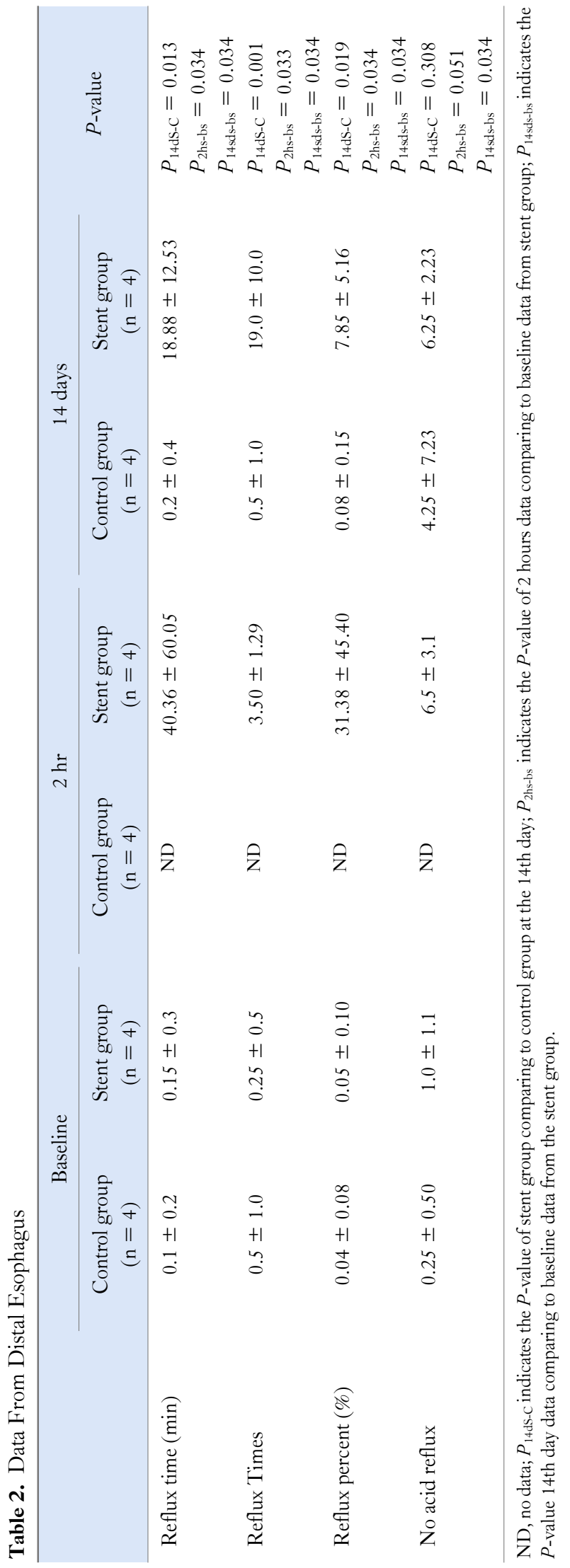



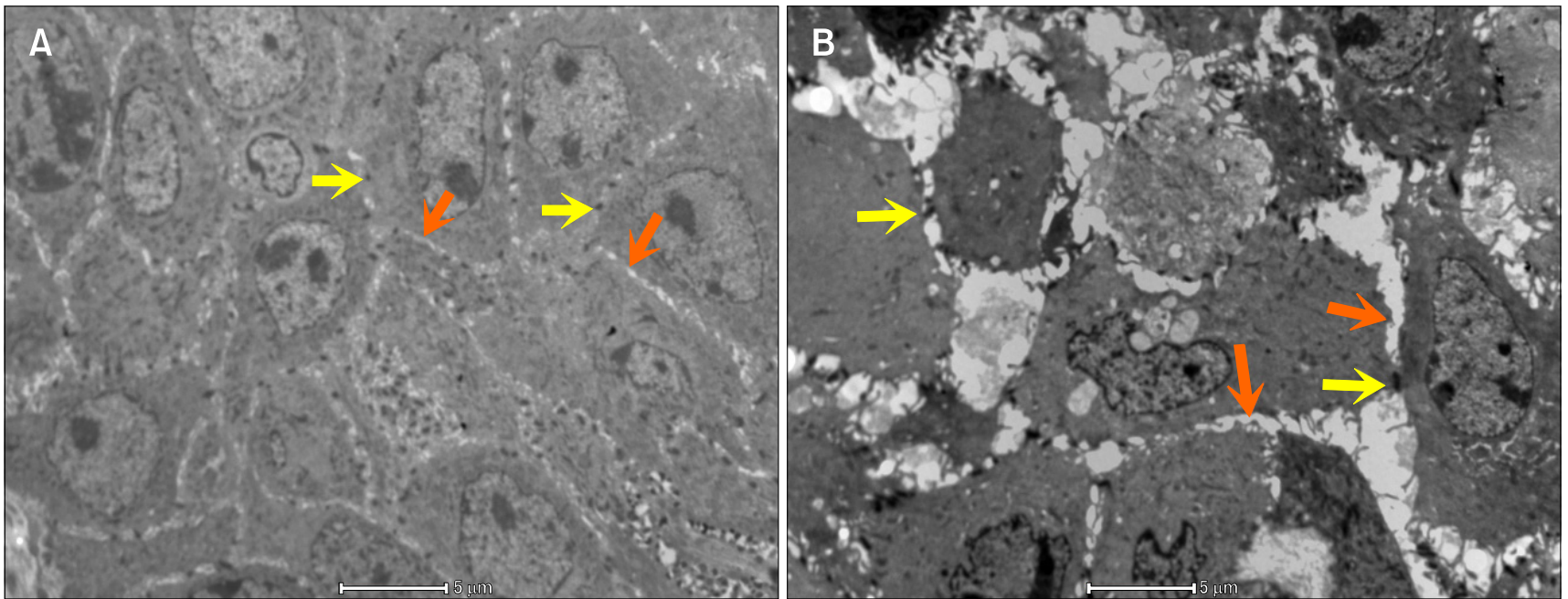

Figure 3. The change of laryngopharyngeal mucosal ultrastructure after stent implant. (A) Intercellular space and desmosomes of laryngopharynx before stent implant: orange arrows indicate intercellular space and yellow arrows indicate desmosomes. (B) Intercellular space and desmosomes of laryngopharynx after stent implant: orange arrows indicate increased intercellular space and yellow arrows indicate decreased desmosomes.

ferent kinds of esophageal gastrointestinal anastomosis. Endogenous GERD model had some advantages for studying the acute pathophysiology of GERD, but it also had key limitations such as the animals needing to have a strong tolerance for the operation and that operators should have a high level of technical skill. In addition, the model animal often died due to various complications as they struggled to survive for a long time, thereby precluding the potential to fully understand the entire development of GERD which is a chronic disease. The advantages of exogenous model included simple operation, high survival rate, and long-term observation. However, there are 2 major limitations of exogenous model: (1) chemicals perfused in esophagus were very different to the complex reflux content in vivo and (2) could not reflect the pathogenesis of reflux disease. Exogenous models are used to study esophageal mucosal damage and defense mechanism. However, the LPR animal model has been reported limitedly. Hu et $\mathrm{al}^{27}$ induced LPR by total cardiomyectomy on albino rabbits in New Zealand to study the association between LPR and GERD. But they did not monitor the objective evidence of reflux, for example the $\mathrm{pH}$ value.

In our study, a custom-designed stent was implanted to destroy kinetic barrier against LPR resulting in stomach contents backflow into oropharynx. We also assessed the reflux by $\mathrm{pH}$ monitoring at the sites of laryngopharynx and distal esophagus and found that this Bama minipig model of LPR was successful. Moreover, there was still reflux 14 days after stent implantation. There is no report about stent implantation to establish LPR model. Our method had advantages including simple operation, high survival rate, less damage, and applicability to study both acute and chronic reflux. So it provided a new method to understand the whole development and pathogenesis of LPR.

In addition, studying mucosal ultrastructure changes may clarify pathogenesis of LPR. ${ }^{27,28}$ Compared with esophageal squamous mucosa, hypopharynx mucosa is easily injured by reflux contents. ${ }^{29}$ In the esophagus, upper limit of tolerance to GER is 50 times per day in the normal physiological condition, but in the hypopharynx, upper limit is generally below 7 times per day. ${ }^{30}$ Many studies found that esophageal intercellular space was increased in GERD and decreased after treatment. ${ }^{19,31-33}$ Significant changes in the intercellular space were also found in the esophageal and laryngeal samples of a rabbit reflux model induced by total cardiomyectomy. ${ }^{27}$ Park et al. found that both LPR patients with and without GERD had a dilated intercellular space in esophageal tissues $(0.95 \pm 0.44 \mu \mathrm{m}$ and $0.61 \pm 0.47 \mu \mathrm{m}$, respectively $)$, compared to the control group $(0.35 \pm 0.27 \mu \mathrm{m}){ }^{20}$ In the present study, we studied the mucosal ultrastructure changes in this LPR model. In the stent group, intercellular space was significantly increased, and the number of desmosomes was significantly decreased, which suggested that LPR damages the laryngopharyngeal mucus.

A Bama minipig model of LPR was established by implanting a custom-designed stent suit. Our model indicates that LPR may damage the laryngopharyngeal mucosal barrier. 


\section{References}

1. Koufman JA, Aviv JE, Casiano RR, Shaw GY. Laryngopharyngeal reflux: position statement of the committee on speech, voice, and swallowing disorders of the American Academy of OtolaryngologyHead and Neck Surgery. Otolaryngol Head Neck Surg 2002;127: $32-35$.

2. Groome M, Cotton JP, Borland M, McLeod S, Johnston DA, Dillon JF. Prevalence of laryngopharyngeal reflux in a population with gastroesophageal reflux. Laryngoscope 2007;117:1424-1428.

3. Hamdan AL, Jabbour J, Barazi R, Korban Z, Azar ST. Prevalence of laryngopharyngeal reflux disease in patients with diabetes mellitus. J Voice 2013;27:495-499.

4. Feng GJ, Zhao LL, Zou N, Liu YL. [Symptom-based relationship between gastroesophageal reflux disease and laryngopharyngeal reflux disease]. Zhonghua Yi Xue Za Zhi 2011;91:2472-2475.

5. Lenderking WR, Hillson E, Crawley JA, Moore D, Berzon R, Pashos CL. The clinical characteristics and impact of laryngopharyngeal reflux disease on health-related quality of life. Value Health 2003;6:560-565.

6. Coca-Pelaz A, Rodrigo JP, Takes RP, et al. Relationship between reflux and laryngeal cancer. Head Neck 2013;35:1814-1818.

7. Komatsu Y, Hoppo T, Jobe BA. Proximal reflux as a cause of adult-onset asthma: the case for hypopharyngeal impedance testing to improve the sensitivity of diagnosis. JAMA Surg 2013;148:50-58.

8. Siupsinskiene N, Adamonis K, Toohill RJ. Quality of life in laryngopharyngeal reflux patients. Laryngoscope 2007;117:480-484.

9. Patcharatrakul T, Gonlachanvit S. Gastroesophageal reflux symptoms in typical and atypical GERD: roles of gastroesophageal acid refluxes and esophageal motility. J Gastroenterol Hepatol 2014;29: 284-290.

10. Chen CL, Yi CH, Liu TT. Relevance of ineffective esophageal motility to secondary peristalsis in patients with gastroesophageal reflux disease. J Gastroenterol Hepatol 2014;29:296-300.

11. Knight RE, Wells JR, Parrish RS. Esophageal dysmotility as an important co-factor in extraesophageal manifestations of gastroesophageal reflux. Laryngoscope 2000;110:1462-1466.

12. Tsutsui H, Manabe N, Uno M, et al. Esophageal motor dysfunction plays a key role in GERD with globus sensation--analysis of factors promoting resistance to PPI therapy. Scand J Gastroenterol 2012;47: 893-899.

13. Vardar R, Sweis R, Anggiansah A, Wong T, Fox MR. Upper esophageal sphincter and esophageal motility in patients with chronic cough and reflux: assessment by high-resolution manometry. Dis Esophagus 2013;26:219-225.

14. Ott C, Ratiu N, Endlicher E, et al. Self-expanding Polyflex plastic stents in esophageal disease: various indications, complications, and outcomes. Surg Endosc 2007;21:889-896.

15. Sreedharan A, Harris K, Crellin A, Forman D, Everett SM. Interventions for dysphagia in oesophageal cancer. Cochrane Database Syst Rev 2009;(4):CD005048.

16. Fukunaga H, Endo W, Ota H, et al. [A clinical case of the esophagogastric malignancy palliated with covered metallic stent with anti-reflux mechanism.] Gan To Kagaku Ryoho 2011;38:2411-2413. [Japanese]
17. Kandulski A, Jechorek D, Caro C, et al. Histomorphological differentiation of non-erosive reflux disease and functional heartburn in patients with PPI-refractory heartburn. Aliment Pharmacol Ther 2013;38:643-651.

18. Zhou LY, Wang Y, Lu JJ, et al. Accuracy of diagnosing gastroesophageal reflux disease by GerdQ, esophageal impedance monitoring and histology. J Dig Dis 2014;15:230-238.

19. Liu CC, Lee JW, Liu TT, Yi CH, Chen CL. Relevance of ultrastructural alterations of intercellular junction morphology in inflamed human esophagus. J Neurogastroenterol Motil 2013;19:324- 331.

20. Park S, Chun HJ, Keum B, et al. An electron microscopic study-correlation of gastroesophageal reflux disease and laryngopharyngeal reflux. Laryngoscope 2010;120:1303-1308.

21. Gill GA, Buda A, Moorghen M, Dettmar PW, Pignatelli M. Characterisation of adherens and tight junctional molecules in normal animal larynx; determining a suitable model for studying molecular abnormalities in human laryngopharyngeal reflux. J Clin Pathol 2005; 58:1265-1270.

22. Ploemen IH, Hirschberg HJ, Kraan H, et al. Minipigs as an animal model for dermal vaccine delivery. Comp Med 2014;64:50-54.

23. Platt SR, Holmes SP, Howerth EW, et al. Development and characterization of a Yucatan miniature biomedical pig permanent middle cerebral artery occlusion stroke model. Exp Transl Stroke Med 2014;6:5.

24. Zeng GQ, Liu R, Liao HX, et al. Single intraperitoneal injection of monocrotaline as a novel large animal model of chronic pulmonary hypertension in Tibet minipigs. PLoS One 2013;8:e78965.

25. Pedersen R, Ingerslev HC, Sturek M, et al. Characterisation of gut microbiota in Ossabaw and Gottingen minipigs as models of obesity and metabolic syndrome. PLoS One 2013;8:e56612.

26. Vodicka P, Smetana K Jr, Dvoránková B, et al. The miniature pig as an animal model in biomedical research. Ann N Y Acad Sci 2005; 1049:161-171.

27. Hu Y, Xu X, Xu L, Lai C, Zhang T. Dilated intercellular space in the larynx and esophagus of a rabbit reflux model. Auris Nasus Larynx 2013;40:379-382.

28. van der Staay FJ, Pouzet B, Mahieu M, Nordquist RE, Schuurman T. The d-amphetamine-treated Gottingen miniature pig: an animal model for assessing behavioral effects of antipsychotics. Psychopharmacology 2009;206:715-729.

29. Powell J, Cocks HC. Mucosal changes in laryngopharyngeal reflux--prevalence, sensitivity, specificity and assessment. Laryngoscope 2013;123:985-991.

30. Johnston N, Dettmar PW, Strugala V, Allen JE, Chan WW. Laryngopharyngeal reflux and GERD. Ann N Y Acad Sci 2013; 1300:71-79.

31. Vaezi MF, Slaughter JC, Smith BS, et al. Dilated intercellular space in chronic laryngitis and gastro-oesophageal reflux disease: at baseline and post-lansoprazole therapy. Aliment Pharmacol Ther 2010; 32:916-924.

32. Xue Y, Zhou LY, Lin SR. Dilated intercellular spaces in gastroesophageal reflux disease patients and the changes of intercellular spaces after omeprazole treatment. Chin Med J 2008;121:12971301.

33. Orlando LA, Orlando RC. Dilated intercellular spaces as a marker of GERD. Curr Gastroenterol Rep 2009;11:190-194. 